\title{
PRIMER REGISTRO DE LIVONECA REDMANI (ISOPODA: CYMOTHOIDAE) COMO PARÁSITO DEL PEZ OLIGOPLITES SAURUS EN LAS AGUAS SOMERAS DE VERACRUZ, MÉXICO
}

\section{First record of Livoneca redmani (Isopoda: Cymothoidae) as parasite of the fish Oligoplites saurus in the shallow waters of Veracruz, Mexico}

\author{
Carlos Manuel Bedia Sánchez ${ }^{1}$, Manuel Ortiz Touzet² ${ }^{2}$ Jonathan Franco López ${ }^{1}$, \\ Edgar Peláez Rodríguez ${ }^{1}$ y José Luis Viveros Legorreta ${ }^{1}$
}

\begin{abstract}
${ }^{1}$ Facultad de Estudios Superiores Iztacala UNAM, Laboratorio de Ecología. bediacharly@yahoo.com.mx.

${ }^{2}$ Facultad de Estudios Superiores Iztacala UNAM, Laboratorio de Crustáceos. ortiztouzet@yahoo.com.
\end{abstract}

\section{RESUMEN}

Se presenta la nueva asociación del isópodo cimotoido Livoneca redmani Leach, 1818, parasitando al pez carángido Oligoplites saurus (Bloch y Schneider, 1801; Pisces: Carangidae), colectado con un chinchorro comercial en Playa Barrancas, Veracruz, México. Se ofrecen además una diagnosis del isópodo, así como tres figuras ilustrativas de este y una del pez parasitado.

Palabras clave: cimotoido, parásito en carángido, Veracruz.

\section{ABSTRACT}

The new association between the cymothoid isopod Livoneca redmani Leach, 1818, parasitizing the carangid fish Oligoplites saurus (Bloch y Schneider, 1801; Pisces: Carangidae), collected with a towing net, at Barrancas Beach, Veracruz, Mexico, is given. A diagnosis of the isopod, as well as three figures of this and one of the host.

Keywords: cymnothoid isopod, carangid fish host, Veracruz.

Los isópodos marinos de las familias Cymothoidae y Aegidae, suelen ser parásitos de peces; sin embargo los del suborden epicaridea lo son únicamente, de otros crustáceos. En ambos casos, producen grandes perjuicios a sus hospederos, cuyas consecuencias son de gran interés al tratarse de especies comerciales. Pueden llegar a afectarles su crecimiento y actividad reproductiva al fijarse sobre la lengua, las branquias, la base de las aletas o sobre la superficie corporal de sus hospederos (Kensley y Schotte, 1989; Bunkley-Williams et al., 1999; Bunkley-Williams, et al., 2006). También los gnátidos, con sus larvas zuphea y praniza, poseen un interesante ciclo de vida, donde la primera depende para subsistir, de un pez hospedero, la segunda es de vida libre y bentónica, que finalmente, se transforma en adulto (Kensley y Schotte, 1989).

Enla región se han publicadonumerosos trabajos de esta temática. La guía de Kensley y Schotte (1989), incluye un total de 28 cimotoidos parásitos con sus respectivos hospederos; Winfield et al. (2002), registran el parasitismo de Anilocra elviae Winfield, Álvarez y Ortiz, 2002, sobre el tiburón Isurus oxyrinchus Rafinesque, 1810, capturado frente a la costa de Veracruz. 
También, Schotte et al., 2009, relacionan un total de ocho aégidos y 13 cimotoidos potencialmente parásitos para el golfo de México. Además, Bonilla Gómez et al. (2014), citan al isópodo Cymothoa excisa Perty, 1833, parasitando al espárido Lagodon rhomboides (Linnaeus, 1766), en el noroeste de la península de Yucatán, mientras que, Chávez et al., (2000) mencionan a Livoneca tenuistylis Richardson, 1912, parasitando una especie de Anchovia, en las aguas venezolanas. Los registros previos de L. redmani, una de las especies parásitas de peces más conocidas en el Atlántico occidental tropical, citan a Scomberomorus maculatus, S. regalis, S. cavalla, Gerres rhombeus y Leiostomus xanthurus como hospederos en sus cámaras branquiales (Kensley y Schotte, 1989).

El material objeto del presente trabajo procede de un estudio que se lleva a cabo en Playa Barrancas, Municipio Antón Lizardo, Veracruz (Fig. 1). La playa las Barrancas se encuentra ubicada frente a la planicie costera del área central del Estado de Veracruz, entre los paralelos $19^{\circ} 00^{\prime} 37^{\prime \prime}$ y $18^{\circ} 59^{\prime}$ y los de latitud Norte y los meridianos $95^{\circ} 58^{\prime} 04^{\prime \prime}$ y $96^{\circ} 00^{\prime \prime}$ de longitud oeste. El hospedero proviene de un lance realizado el 19 de septiembre de 2016, con un chinchorro playero de $800 \mathrm{~m}$ de largo por $4 \mathrm{~m}$ de altura, con luz de malla de $4.5 \mathrm{~cm}$ y copo de 15 metros. El material colectado fue fijado con formolina al $10 \%$, y trasladado al laboratorio, donde se realizaron las identificaciones pertinentes, se registró la longitud patrón (Lp) y peso total (W) tanto del hospedero como del parásito. Para la determinación de la madurez sexual del hospedero, se empleó el sistema propuesto por Nikolsky (1963).

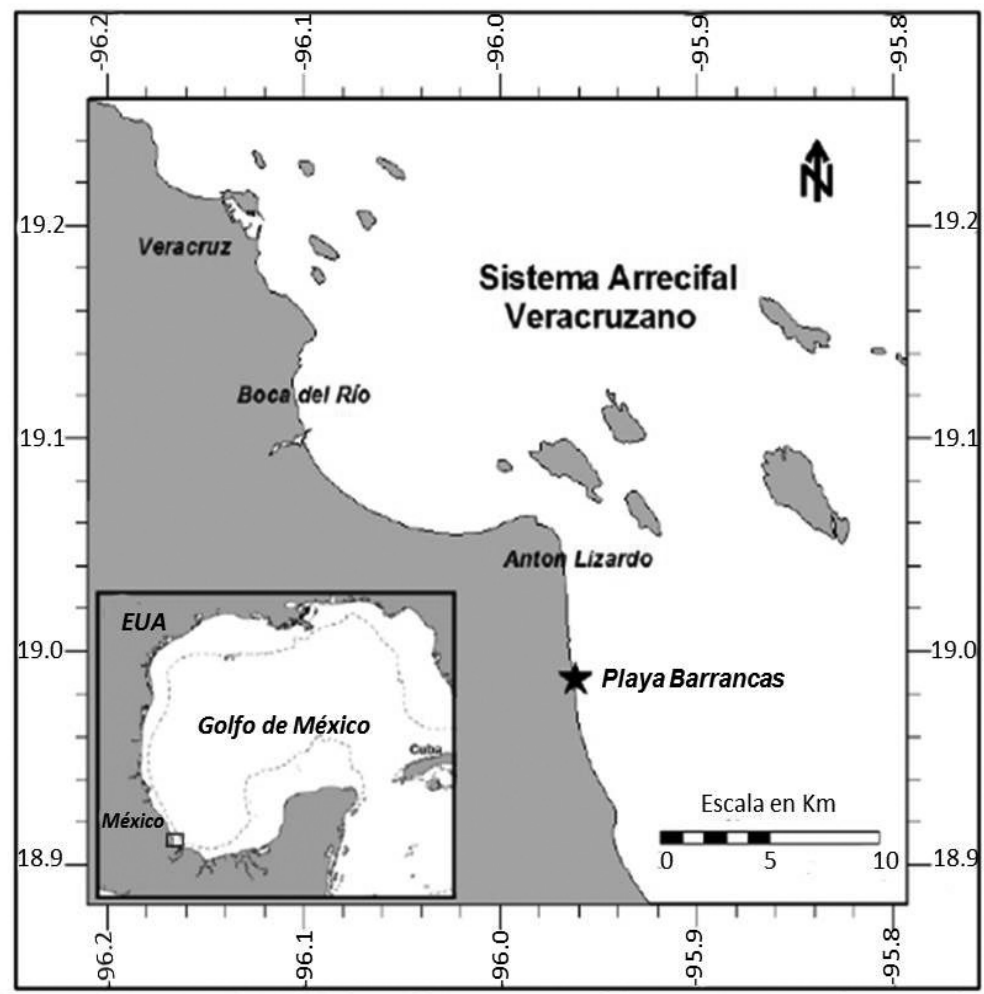

Figura 1. Ubicación de la localidad de colecta. 


\section{TAXONOMÍA}

Orden ISOPODA Latreille, 1817

Suborden CYMOTHOIDA Wägele, 1989

Superfamilia CYMOTHOOIDEA Leach, 1814

Familia CYMOTHOIDAE Leach, 1818

Género Livoneca Leach, 1818

(ortografía según el CINZ, 3ra. Edición; Bruce, 1990)

Livoneca redmanii Leach, 1818

(Fig. 2)

Material estudiado: hembra ovígera; longitud patrón $2.1 \mathrm{~cm}$; ancho $1.2 \mathrm{~cm}$; peso $0.66 \mathrm{gr}$; diámetro promedio de los huevos $0.8 \mathrm{~mm}$; localizada fijada en la zona dorsolateral y posterior de la cabeza del hospedero.

Diagnosis. Cabeza más corta que ancha; cabeza hundida ligeramente en el primer segmento pereonal; cuerpo liso y asimétrico; coxas visibles en vista dorsal; telson más corto que ancho; segmentos pleonales curvados hacia delante; pleópodos sobresalen por debajo del borde posterior telsónico; endopodito del urópodo dos veces más largo que ancho.

Distribución. Atlántico occidental: Bermudas, New Jersey, Florida, Cuba, Jamaica, Campeche (México) Panamá, Venezuela y Colombia. Atlántico oriental: costas de África tropical. (Kensley y Schotte, 1989; Vilchez et al., 2008); Schotte et al., 2009: Boyko et al., 2018).

Características del hospedero. Oligoplites saurus (Bloch y Schneider, 1801): macho en estadio III de madurez sexual; longitud patrón de 20.2 cm; peso 95.86 gr (Fig. 3).

Con esta publicación se hace el primer registro de L. redmani como ectoparásito del pez Oligoplites saurus para las aguas de playa Barrancas, en Veracruz, y para todo el golfo de México.
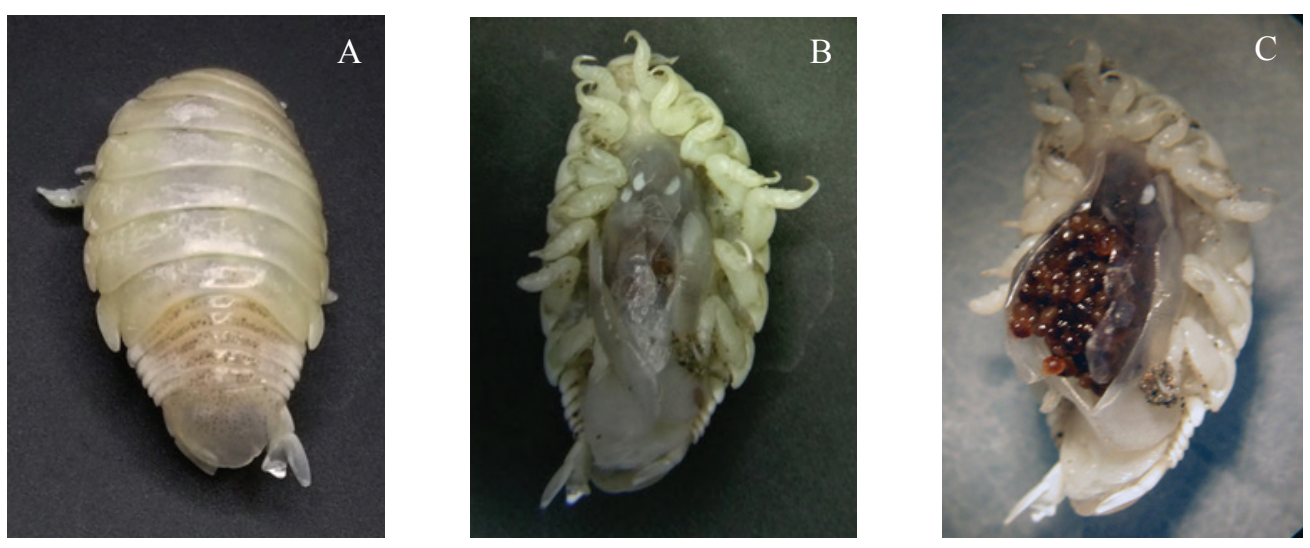

Figura 2. Diferentes vistas del ectoparásito, Livoneca redmani. A, vista dorsal. B, vista ventral. C, vista ventral presentando el marsupio abierto con sus huevos. 


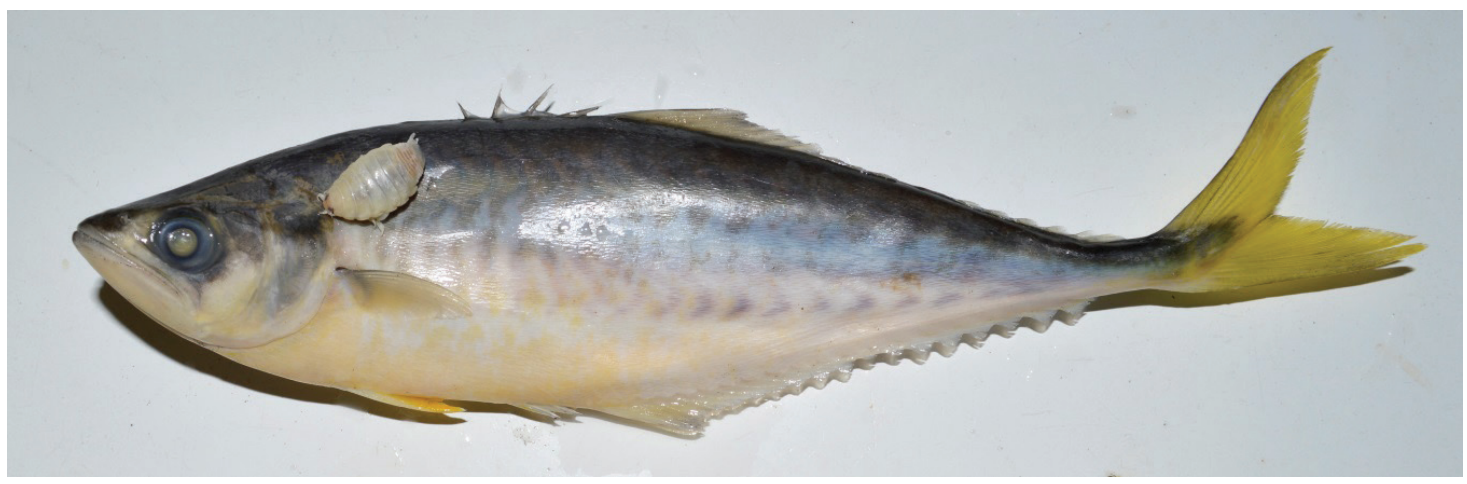

Figura 3. Vista lateral del pez hospedero Oligoplites saurus, con el ectoparásito L. redmani "in situ".

\section{AGRADECIMIENTOS}

Los autores extienden un cordial agradecimiento a la División de Investigación de la FES-Iztacala, por apoyar parte del trabajo realizado y al Técnico Pesquero Tomas Corro Ferreira, del Centro de Estudios Tecnológicos del Mar, No. 7, Veracruz, por su apoyo en la colecta del material biológico.

\section{LITERATURA CITADA}

Bonilla-Gómez, J. L., A. Ramírez-Rojas, M. Badillo-Alemán y J. Chiappa- Carrara. 2014. Nuevo registro de Lagodon romboides (Pisciformes: Sparidae) como hospedero de Cymothoa excisa (Isopoda, Cymothoidae) en la costa noroeste de la península de Yucatán. Revista mexicana de Biodiversidad, 85: 634-637.

Boyko, C. B., N. L. Bruce, K. A. Hadfield, K. L. Merrin, Y. Ota, G. C. B. Poore, S. Taiti, M. Schotte y G. D. F. Wilson. (Eds) 2008 onwards. World Marine, Freshwater and Terrestrial isopod Crustaceans database, consultado en htpp://www. marineespecies.org/ isopoda on 2018-01-12.

Bruce, N. L. 1990. The genera Catoessa, Elthusa, Enispa, Ichthyocenus, Idusa, Livoneca and Norileca n. gen. (Isopoda, Cymothoidae), crustaceans parasites of marine fishes, with description of Eastern Australian species. Records of the Australian Museum, 42 (3): 247-300.

Bunkley-Williams, L., E. H. Williams, Jr. y J. Garzón-Ferreira. 1999. Some isopod and copepod parasites (Crustacea) of Colombian marine fishes. Caribbean Journal of Science, 35: 311-314.

Bunkley-Williams, L., E. H. Williams, Jr. y A. K. M. Bashirullah. 2006. Isopods (Isopoda: Aegidae, Cymothoidae, Gnathiidae) associated with Venezuelan marine fishes (Elasmobranchii, Actinopterygii). Revista de Biología Tropical, 54: 175-188.

Chávez, R., J. M. Delgado, M. Paredes y E. Weir. 2000. Primer registro de Lironeca tenuistylis (Richardson, 1912) (Isopoda: Cymothoidae) para Venezuela. Boletín del Centro de Investigaciones Biológicas, Universidad de Zulia, 34 (3): 423-428. 
Kensley, B. y M. Schotte. 1989. Marine isopod crustaceans of the Caribbean. Smithsonian Institution Press, Washington, D. C. 308 pp.

Nikolsky, G. 1963. The Ecology of fishes. Academic Press Inc. (London), Ltd. Sixth Printing USA. 1976:145-225.

Schotte M., J. C. Markham y G. D. F. Wilson. 2009. Isopoda (crustacean) of the Gulf of Mexico. In: Felder D. L., Camp D. K., editors. Gulf of Mexico Origins, Waters and Biota.Vol.1, Biodiversity. Texas A\&M Univ. Press, USA. pp. 973-986.

Vilches, I., S. Ramírez y J. G. Delgado. 2008. Primer registro de Livoneca redmanii Leach, 1818 (Isopoda: Cymothoidae) para el Lago Maracaibo, Venezuela. Boletín del Centro de Investigaciones Biológicas, Universidad de Zulia, 42 (2): 269-273.

Winfield, I., Álvarez, F. y M. Ortiz. 2002. A new species of Anilocra (Crustacea: Isopoda: Cymothoidae), ectoparasite on the mako shark Isurus oxyrinchus. Proceedings of the Biological Society of Washington, 115 (1): 148-152.

[Recibido: 06 de abril, 2018. Aceptado para publicación: 24 de abril, 2018] 